\title{
A Transform Space Filtered, Wide Frequency-Range Implementation of the Parabolic Equation Method
}

\author{
Steve J. Salamon \\ Radio Transport, Telstra, and \\ School of Electrical \& Electronic \\ Engineering, University \\ of Adelaide, SA 5005, Australia
}

\author{
Hedley J. Hansen \\ RFT Group EWRD, DSTO \\ PO Box 1500, Edinburgh, \\ SA 5111, Australia
}

\author{
Derek Abbott \\ School of Electrical \& Electronic \\ Engineering \\ University of Adelaide \\ SA 5005, Australia
}

\begin{abstract}
Modelling of point-to-point radio propagation over terrain is of interest in the design of radio systems working over obstructed radio paths, or which may be subject to sub-refractive fading, or in estimating the visibility of radar targets close to terrain. Practical implementation of Fourier split-step PEM to provide accurate prediction of field-strength deep into the terrain diffraction region, over a wide frequency range, faces significant challenges. At high frequencies a large transform size is required, and at low frequencies the artificial upper boundary must be sufficiently high and the absorber layer sufficiently thick, to prevent spurious reflections from the upper boundary interfering with the weak terrain diffracted field. An adaptation to the PE method is described, and tested for the canonical problems of wedge and smooth-Earth diffraction over the frequency range of $10 \mathrm{MHz}$ to $100 \mathrm{GHz}$, and compared with path measurements from $150 \mathrm{MHz}$ to $1.5 \mathrm{GHz}$.
\end{abstract}

Keywords- parabolic equation method; radio propagation; terrain diffraction; sub-refraction.

\section{INTRODUCTION}

The Fourier split-step approach is a practical solution to the parabolic wave equation for modelling point-to-point radio propagation over terrain, as it provides a marching solution along the path, which can have a range-step that is large compared to the wavelength.

Simplification of the problem is possible at very high frequencies and at small angles compared to the Brewster angle, because the Dirichlet boundary condition (reflection coefficient $=-1$ ) is a reasonably accurate representation of the ground surface. This boundary condition is automatically satisfied by choosing the sine transform, due to its odd symmetry, as the form of Fourier transform used in the Fourier split-step Parabolic Equation Method (PEM).

Although the physical problem of terrestrial propagation has no upper bound, an artificial upper boundary is required for practical analysis, and various techniques have been employed to ensure that fields propagating upwards out of the height range of interest are not reflected back into it.

An absorber layer can be created by attenuating the highest field samples with an appropriate window function $[1,2]$, which is most effective at small angles to the horizontal [2]. If small step lengths are used, the specific attenuation ( $\mathrm{dB}$ per $\mathrm{km}$ ) can rise rapidly above the bottom of the absorber layer, so care is required at low frequencies to ensure this does not result in reflection near the bottom of the absorber. For problems involving larger angles, the Perfectly Matched Layer [2] is an effective technique.

The approach investigated replaces the usual approximation of an artificial absorbing upper boundary, with filtering in transform (angle) space, not just as often applied in the upper half of that space $[1,2]$, but filtering applied over the whole of the transform space.

\section{TRANSFORM SPACE FILTERING IN FOURIER SPLIT-STEP PEM}

The impact of transform space filtering on the Fourier splitstep solution to the parabolic equation, can be explored by considering the equivalent to this filtering approximation, when applied to the Fresnel-Kirchoff theory of diffraction, due to the equivalence between these two approaches [2]. We consider initially the case of free-space propagation, and then knife-edge diffraction.

\section{A. Free-space propagation}

Consider the complex Fresnel Integral $F_{c}(v)$, where $v$ is the diffraction parameter:

$$
F_{c}(v)=\int_{0}^{v} \exp \left(j \frac{\pi s^{2}}{2}\right) \mathrm{d} s=C(v)+j S(v),
$$

which can be expressed in terms of two real integrals, a cosine integral $C(v)$ and a sine integral $S(v)$. Free-space propagation is then represented by $F_{c}(\infty)-F_{c}(-\infty)$, shown as a straight line in Figure 1.

Where an unobstructed ray-path exists between transmitter and receiver, the filtering investigated in this paper is Gaussian with respect to $v$, according to real filter parameter $c$, to obtain an approximation $F_{a}(v)$ to the complex Fresnel integral:

$$
F_{a}(v)=\int_{0}^{v} \exp \left((j-c) \frac{\pi s^{2}}{2}\right) \mathrm{d} s=C_{a}(v)+j S_{a}(v)
$$


For values of the filter parameter $c$ less than 0.1 , the amplitude error introduced by the approximation (2) is approximately $c^{1.5} \mathrm{~dB}$ for free-space propagation, where the relative field strength is represented by $F_{a}(\infty)-F_{a}(-\infty)$. Phase error is approximately $c / 2$ radians, so the error introduced at low values of $c$ is predominantly phase error.

The approximation (2) converges to its approximate solution much more quickly than the very slowly converging Fresnel integral (1). This is demonstrated by plotting (1) and (2) in the form of a Cornu spiral. Figure 1 shows this for $F_{c}(v)$, and $F_{a}(v)$ with $c=0.1$.

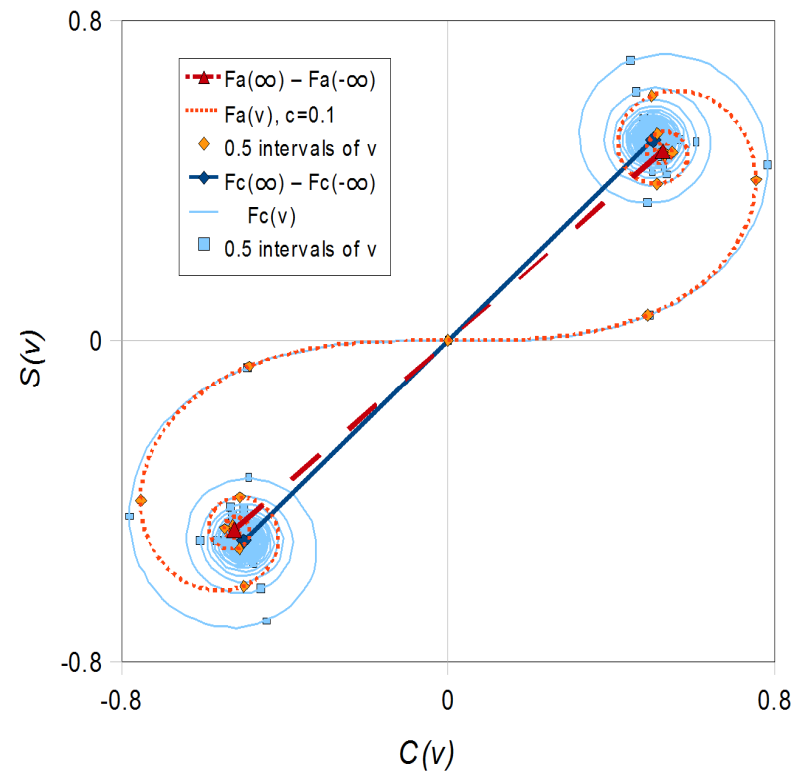

Figure 1. Complex Fourier integral $F_{c}(v)$, and approximation $F_{a}(v)$ with filter constant $c=0.1$. The resultant field-strength for a free-space path is indicated by the lines $F_{c}(\infty)-F_{c}(-\infty)$ and $F_{a}(\infty)-F_{a}(-\infty)$.

Neglecting initially atmospheric refraction, Earth curvature and terrain height variation, the implementation of the Fourier split-step method [1], is given by

$$
\psi(x+\Delta x, z)=\mathfrak{I}^{-1}\left\{e^{j \Delta x\left(\sqrt{k^{2}-p^{2}}-k\right)} \mathfrak{I}\{\psi(x, z)\}\right\},
$$

where the field-points $\psi(x+\Delta x, z)$, at distance $x+\Delta x$ along the path are obtained from the field-points $\psi(x, z)$, at distance $x$ along the path, by transforming to angle space with the Fourier transform, then applying the exponential term, which is the free-space propagator, and then applying the inverse transform. The transform variable is $p=k \cdot \sin \theta$, where $k$ is the wave number, and $\theta$ is the angle relative to the horizontal. For small $\theta$, the equivalent formulation to (2) is approximately implemented by the following:

$$
\psi(x+\Delta x, z)=\mathfrak{I}^{-1}\left\{e^{(j-c) \Delta x\left(\sqrt{k^{2}-p^{2}}-k\right)} \mathfrak{I}\{\psi(x, z)\}\right\}
$$

The Gaussian filtering resulting from replacing $j$ in (3) with $(j-c)$ in (4), applied at each step of the PEM analysis, accumulates over the end-to-end path to produce a ray-path dependent Gaussian filter, which is equivalent to the Gaussian filtering term $\exp \left(-c \pi s^{2} / 2\right)$ in (2).

\section{B. Knife-edge diffraction}

When a knife-edge obstruction blocks the ray-path between transmitter and receiver, the most significant field-points above the knife-edge are those immediately above it, and contributions from these to the received field would incur filtering loss if the approximation of (2) was used to evaluate the received field. This can be avoided by employing a coordinate system where the field is steered at the knife-edge, such that in the new coordinate system, the knife-edge is at grazing incidence.

This is analogous to the usual field steering used in the terrain flattening algorithm for Fourier split-step PEM, except that the field steering here is according to the bends in the traced ray, where it has to bend around terrain in order to reach the receiver, instead of steering the analysis according to the bends in the terrain itself. The piece-wise linear shift-map [3] is used in this analysis:

$$
\psi_{2}\left(x_{1,2}, z\right)=\psi_{1}\left(x_{1,2}, z\right) e^{j k z\left[\sin \beta_{1}-\sin \beta_{2}\right]},
$$

except that in this application the angles $\beta_{1}$ and $\beta_{2}$ are the angles to the horizontal of the traced rays from transmitter to knife-edge top $\left(\beta_{1}\right)$, and from knife-edge top to receiver $\left(\beta_{2}\right)$.

This approach applied to knife-edge diffraction, that is, the approximate complex Fresnel integral of (2) for the case of zero diffraction angle, combined with the piece-wise linear shift-map of (5) with the angles $\beta_{1}$ and $\beta_{2}$ corresponding to actual diffraction angle $\theta=\beta_{1}-\beta_{2}$, using the approximation $k z\left[\sin \beta_{1}-\sin \beta_{2}\right] \approx s \pi v$ for $v \geq 0$, leads to the following approximation:

$$
F_{a}(\infty)-F_{a}(v) \approx \int_{0}^{\infty} \exp \left((j-c) \frac{\pi s^{2}}{2}-j \pi v s\right) \mathrm{d} s .
$$

The maximum amplitude error, using (6) to estimate knifeedge diffraction loss, for example using $c=0.2$, is $0.12 \mathrm{~dB}$, occurring at $v=0.8$. The purpose of the approximations to the Fresnel integral of (2) and (6) is to obtain a rapidly converging expression, so that in practical evaluation, the integral to infinity can be truncated well before infinity, with negligible additional error.

\section{Transform Size}

The purpose of the approximation to the PEM solution of (4) is analogous to the purpose of the approximation to the Fresnel integral in (2) or (6); the aim is to allow evaluation over a limited height range, without the need of an artificial absorbing upper boundary. This eliminates the need to adapt 
the characteristics of this upper boundary to the frequency, when operating over a very wide frequency range.

The maximum vertical deviation $z_{\max }$ of field points from the traced ray-path, required for accurate implementation of this method, can be estimated by considering, assuming no atmospheric refraction, a ray from the transmitter to a midpath point deviated $z_{\max }$ above or below the direct ray-line, and then to the receiver. For a path length $d$, the phase delay $\Delta \varphi$ of this path with respect to the direct path is approximately given by

$$
\Delta \phi \approx \frac{2 k z_{\max }^{2}}{d}
$$

and the Gaussian filtering term of (4), accumulated over the end-to-end path, can be expressed as a window function $W(\Delta \varphi)$ :

$$
W(\Delta \phi)=e^{-c \Delta \phi}
$$

Choosing a suitably small value of this function at the point where the analysis is truncated, for example $W(\Delta \varphi)=10^{-7}$, we have

$$
z_{\max }=\sqrt{\frac{1.28 \lambda d}{c}}
$$

The maximum vertical spacing of field-points in the analysis is determined by the maximum angle $\theta_{\max }$ through which the field will be steered, in order to avoid signal loss in grating lobes [3]:

$$
\Delta z<\frac{\lambda}{1+\sin \theta_{\max }}
$$

The minimum transform size, $N_{\min }$, is then given by:

$$
N_{\text {min }}=\left(1+\sin \theta_{\max }\right) \sqrt{\frac{5.12 d}{c \lambda}} .
$$

At high frequencies, $N_{\min }$ given by (11) could be significantly smaller than the transform size required by conventional Fourier split-step PEM, which must include all field-points from ground level up to above the height of interest, as well as satisfying (10).

\section{Ground Reflection}

The boundary condition at the ground surface is not automatically satisfied by the Fourier transform in (4) when the Fast Fourier Transform (FFT) is used directly as the practical implementation of the transform, so the boundary condition is instead satisfied by either by manipulating the field-points at the beginning of the step, $\psi(x, z)$; or by manipulating the fieldpoints at the end of the step $\psi(x+\Delta x, z)$.

If the ground at the beginning of the step is closer to the traced ray-path than at the end, the above-ground field-points $\psi\left(x, z_{\mathrm{g}}+h\right)$, where $z_{\mathrm{g}}$ is the ground height relative to the tracedray, and $h$ is the height of the field-point above ground, are copied to the same height below-ground, after being multiplied by reflection coefficient $R$ and a phase and amplitude correction term $\exp \left[(j-c) \varphi_{\beta}\right]$. The phase correction $\varphi_{\beta}$ accounts for additional phase delay in the reflected path resulting from the downward slope of the ground with respect to the tracedray. If the corresponding direct ray-path is parallel to the traced-ray, or $p=0$ in (4), the additional reflected path phase delay $\varphi_{\beta}$ incurred due to the ground slope $\beta$ given by

$$
\phi_{\beta}=k\left(\sqrt{4 h^{2} \cos ^{4} \beta+(h \sin (2 \beta)+\Delta x)^{2}}-\Delta x\right) .
$$

The expression (12) is approximate for direct rays not parallel to the traced-ray, but these rays and their reflected rays are attenuated by the filtering applied by (4), so the small error in the reflected phase may be insignificant. The image points added to $\psi(\mathrm{x}, \mathrm{z})$ before evaluation of (4) are then given by

$$
\psi\left(x, z_{g}-h\right)=R \psi\left(x, z_{g}+h\right) \exp \left((j-c) \phi_{\beta}\right)
$$

The addition of image field-points using (13) is truncated at $z_{\mathrm{g}}-h=-z_{\max }$. If the field-points at the bottom edge of $\psi(x, z)$ were not attenuated by the filtering of this method, this truncation would result in a spurious aliased field appearing at the top of $\psi(x+\Delta x, z)$, if $z_{\mathrm{g}}$ is negative (ground level below the middle of the transform space), but this effect does not occur when the ground reflection is implemented at the end of the step, so reflection at the end of the step is preferred for ground parallel to the traced ray-path.

If the ground is parallel to, or closer to the traced ray-path at the end of the step than at the beginning, the ground reflection is implemented at the end of the step in a manner similar to (13):

$$
\begin{aligned}
& \psi\left(x+\Delta x, z_{g}+h\right)=\psi\left(x+\Delta x, z_{g}+h\right) \\
& \quad+R \psi\left(x+\Delta x, z_{g}-h\right) \exp \left((j-c) \phi_{\beta}\right),
\end{aligned}
$$

for those values of $\psi\left(x+\Delta x, z_{\mathrm{g}}+h\right)$ where the image field point $\psi\left(x+\Delta x, z_{\mathrm{g}}-h\right)$ exists. The additional reflected path phase delay $\varphi_{\beta}$ incurred due to the ground slope $\beta$ (this time defined as the upward slope towards the end of the step, so still positive) is again given by (12).

\section{E. Terrain Obstruction and Refractivity Gradient}

Ray-tracing is used to find the shortest phase-length raypath from transmitter to receiver, but if this does not exist due to terrain obstruction, the lowest angle ray from the transmitter 
to a point tangent to the terrain (the transmitter horizon) is taken as the first part of the shortest ray-traced path. From this tangent point, or horizon, the process is repeated until the receive antenna is reached. At intervening horizon points, the traced-ray, which is the centre datum of this analysis method, may change direction. This is allowed for by using the piecewise linear shift-map [3], but with $\beta_{1}$ and $\beta_{2}$ being the angles to the horizontal of the traced-ray either side of the change in direction.

For consistency with the rest of the analysis, rather than using the original form of the shift-map (5), it might be expected that the filter constant $c$ would be included:

$$
\psi_{2}\left(x_{1,2}, z\right)=\psi_{1}\left(x_{1,2}, z\right) e^{(j-c) k z\left[\sin \beta_{1}-\sin \beta_{2}\right]},
$$

though testing with wedge diffraction paths, measured paths, and smooth-Earth paths indicated that this results in mean over-prediction of path loss for $c>0.1$. Omitting $c$ in (15) appeared to result in mean under-prediction of path loss, but the following form of the shift-map was found to provide reasonable accuracy for $0.025 \leq c \leq 0.8$; this includes phaseshift for modified refractivity $m(x, z)$, with $m_{0}(x)$ being the modified refractivity at distance $x$ along the traced ray:

$$
\begin{aligned}
& \psi_{2}\left(x_{1,2}, z\right)=\psi_{1}\left(x_{1,2}, z\right) . \\
& e^{(j-0.3 \sqrt{c}) k\left\{z\left[\sin \beta_{1}-\sin \beta_{2}\right]+m(x, z)-m_{0}(x)\right\}+j k m_{0}(x)}
\end{aligned}
$$

The approximation to the PEM described by (4), (9), (12), (13), (14) and (16) was used for the testing which follows in Section III.

\section{Testing The Transform SPACE FILTERED PEM}

Initial testing was conducted using the canonical models of wedge diffraction and smooth-sphere diffraction, over a very wide frequency range, assuming the Dirichlet boundary condition (horizontal polarisation and infinite conductivity) which is a reasonable approximation for real paths at any polarisation at grazing angles, small compared to the Brewster angle. A dataset of accurately calibrated VHF and UHF obstructed paths, without tree cover, [4] was also tested.

\section{A. Wedge Diffraction}

The example presented here is a $10 \mathrm{~km}$ path, with the PEM in $1 \mathrm{~km}$ steps, having terrain height rising linearly from zero at each terminal to $400 \mathrm{~m}$ at the central $5 \mathrm{~km}$ point. Antenna heights are $400 \mathrm{~m}$ at the transmitter and $100 \mathrm{~m}$ at the receiver, although the same results from the PEM analysis were obtained with these heights swapped. The reference method was UTD wedge diffraction [5], with calculated diffraction loss (loss in excess of free-space) ranging from $22.8 \mathrm{~dB}$ at $10 \mathrm{MHz}$ to 59.6 $\mathrm{dB}$ at $100 \mathrm{GHz}$.

Accuracy of the filtered PEM analysis, for various values of the filter parameter $c$, is demonstrated by Figure 2 .



Figure 2. Accuracy of the transform-space filtered PEM with various values of the filter parameter $c$, for a $10 \mathrm{~km}$ wedge path in $1 \mathrm{~km}$ steps, with apex 400 metres high, and antenna heights $400 \mathrm{~m}$ and $100 \mathrm{~m}$.

While $c$ values of 0.1 or less provided the most accurate results for frequencies of $1 \mathrm{GHz}$ or higher as expected, higher values of $c$ were more accurate at VHF frequencies.

\section{B. Smooth-Earth Diffraction}

Figure 3 demonstrates the accuracy in analysing a $50 \mathrm{~km}$ smooth-Earth path, in 50 metre steps, with 50 metre antenna heights, and effective Earth radius of $2800 \mathrm{~km}$ (sub-refraction $+200 \mathrm{~N}$-units/km).



Figure 3. Accuracy of the filtered PEM for a $50 \mathrm{~km}$ smooth spherical-Earth path, in $50 \mathrm{~m}$ steps, with effective radius $2800 \mathrm{~km}$, and antenna heights of 50 metres. Reference method is the smooth-Earth diffraction model of Recommendation ITU-R P.526 [5], with path losses from free-space plus 41 $\mathrm{dB}$ at $10 \mathrm{MHz}$, to free-space plus $161 \mathrm{~dB}$ at $100 \mathrm{GHz}$. 


\section{Measured VHF and UHF Paths}

A dataset of 450 carefully calibrated measurements on 61 paths, without tree cover, in South Australia and Northern Territory, were used test the accuracy of this method. It is essentially the same dataset used previously [4], except that the original topographic map path profiles have been replaced by profiles with $50 \mathrm{~m}$ spacing between points, generated from Shuttle Radar Topography Mission data. Three paths in the original dataset have been excluded; one was found to be a duplicate, and two both less than $5 \mathrm{~km}$ long were significant outliers for all 2D models tested.

In addition to the method of this paper, results for conventional Fourier split-step PEM analysis, using the sine transform, and cosine taper absorber applied to the the upper half of the field-points [3], and also a largely empirical "Method for a general terrestrial path" recently developed by ITU-R Study-Group 3 [5], are presented in Table 1.

TABLE I. ACCURACY COMPARISON BETWEEN FILTERED AND CONVENTIONAL FOURIER SPLIT-STEP PEM, AND A RECENT ITU-R GENERAL TERRAIN DIFFRACTION MODEL, FOR 450 PATH MEASUREMENTS AT $150 \mathrm{MHZ}$ $509 \mathrm{MHz}$, AND $1481 \mathrm{MHz}$.

\begin{tabular}{|c|c|c|}
\hline \multicolumn{3}{|c|}{ Diffraction Loss Prediction Error } \\
\hline Analysis Method & Mean Error & Standard Deviation \\
\hline $\begin{array}{c}\text { Transform filtered PEM (this } \\
\text { paper) with } c=0.4\end{array}$ & $-0.9 \mathrm{~dB}$ & $3.5 \mathrm{~dB}$ \\
\hline $\begin{array}{c}\text { Sine transform Fourier split- } \\
\text { step PEM [3] }\end{array}$ & $-2.9 \mathrm{~dB}$ & $4.1 \mathrm{~dB}$ \\
\hline Rec. ITU-R P.526-12 $\S 4.5[5]$ & $-1.1 \mathrm{~dB}$ & $4.6 \mathrm{~dB}$ \\
\hline
\end{tabular}

\section{EXISTING PROPAGATION TOOLS}

Software implementations of the conventional PEM solution [1, 2, 3] are available [6, 7], which provide a convenient user interface for typical radar applications, but for propagation research, or special applications beyond normal radar parameter ranges, it may be better to write software specific to the task. Modern scientific programming languages make this relatively straight-forward, except for the problem of finding the right configuration for the artificial upper boundary, and the appropriate transform space low-pass filtering and antenna patterns, to avoid spurious results; this can be rather empirical.

This problem is eliminated by the method of this paper, although it introduces the possibility that signals following raypaths at angles significantly divergent from the angle of the shortest ray-path may be attenuated; however this is probably not important for estimating field-strength deep in the shadow region.

For example, there may be a need to estimate path-loss, or propagation factor, between a high power transmitter and a sensitive deep-space receiver or radio-telescope. This may involve propagation factors below those that the off-the-shelf tool was designed to handle. Figure 3 demonstrates reasonable accuracy in the estimation of propagation factor over smooth terrain, down to as low as $161 \mathrm{~dB}$ below free-space, using the method of this paper.

\section{CONCLUSIONS}

An adaptation of the PEM solution has been described, which provides stable results over a wide frequency range, and eliminates the requirement for an artificial upper boundary. Its accuracy has been tested for two dimensional terrain diffraction problems with linear refractivity gradients.

Three dimensional terrain diffraction problems, or two dimensional problems with non-linear refractivity gradents, may have multiple transmitter to receiver ray-paths, some of which may be attenuated by the filtering in this PEM adaptation. Further work should consider techniques to identify and compensate for this attenuation.

\section{REFERENCES}

[1] Barrios, A.E., "A terrain parabolic equation model for propagation in the troposphere," IEEE Trans. Antennas and Propagation, 42(1), pp. 90-98, January, 1994.

[2] Levy, M., Parabolic Equation Methods for Electromagnetic Wave Propagation, IET Electromagnetic Waves Series, 45, 2000.

[3] Donohue, D.J., Kuttler, J.R., "Propagation modelling over terrain using the parabolic wave equation," IEEE Trans. Antennas and Propagation, 48(2), pp. 260-277, February, 2000.

[4] Salamon, S.J., Wilson, C., "Reducing discontinuity in practical prediction of diffraction loss in irregular terrain," Proc. ISART/Climdiff 2008, pp.259-266, June 2008.

[5] Recommendation ITU-R P.526-12, "Propagation by diffraction," Geneva February 2012.

[6] Paterson, W.L., "Advanced Refractive Effects Prediction System (AREPS)," Proc. 2007 IEEE Radar Conference, pp.891-895, April 2007.

[7] Brookner, E., Cornely, P.-R, Lok, Y.F., "AREPS and TEMPER getting familiar with these powerful propagation software tools," Proc. 2007 IEEE Radar Conference, pp.1034-1043, April 2007. 